\title{
Taking ownership of safety. What are the active ingredients of safety coaching and how do they impact safety outcomes in critical offshore working environments?
}

Article

Accepted Version

Krauesslar, V., Avery, R. E. and Passmore, J. (2015) Taking ownership of safety. What are the active ingredients of safety coaching and how do they impact safety outcomes in critical offshore working environments? International Journal of Occupational Safety and Ergonomics, 21 (1). pp. 39-46. ISSN 1080-3548 doi:

https://doi.org/10.1080/10803548.2015.1017941 Available at https://centaur.reading.ac.uk/81941/

It is advisable to refer to the publisher's version if you intend to cite from the work. See Guidance on citing.

To link to this article DOI: http://dx.doi.org/10.1080/10803548.2015.1017941

Publisher: Taylor \& Francis

All outputs in CentAUR are protected by Intellectual Property Rights law, including copyright law. Copyright and IPR is retained by the creators or other copyright holders. Terms and conditions for use of this material are defined in the End User Agreement. 


\section{www.reading.ac.uk/centaur}

\section{CentAUR}

Central Archive at the University of Reading

Reading's research outputs online 


\title{
Taking Ownership of Safety. What are the active ingredients of safety coaching and how do they impact safety outcomes in critical offshore working environments?
}

Victoria Krauesslar, Rachel E. Avery \& Jonathan Passmore

\begin{abstract}
Safety coaching interventions have become a common feature in the safety critical offshore working environments of the North Sea. Whilst the beneficial impact of coaching as an organisational tool has been evidenced, there remains a question specifically over the use of safety coaching and its impact on behavioural change and producing safe working practices. A series of 24 semi-structured interviews were conducted with three groups of experts in the offshore industry: safety coaches, offshore managers and HSE directors. Using a thematic analysis approach, several significant themes were identified across the three expert groups including connecting with and creating safety ownership in the individual, personal significance and humanisation, ingraining safety and assessing and measuring a safety coach's competence. Results suggests clear utility of safety coaching when applied by safety coaches with appropriate coach training and understanding of safety issues in an offshore environment. The current work has found that the use of safety coaching in the safety critical offshore oil and gas industry is a powerful tool in managing and promoting a culture of safety and care.
\end{abstract}

Keywords:

Safety-Coaching, Environmental, High-Risk, Oil-and-Gas-Industries; Safety-Critical.

\section{Introduction}

Coaching is defined as a collaborative relationship focused on valued goal attainment aimed at development of skills/performance [1]. A recent systematic review found that coaching leads to greater training success, employee well-being, goal commitment and achievement as well as organisational productivity [1]. Other research has shown that coaching intervention combined with traditional training led to significant increases in productivity levels relative to training alone [2]. One 
type of coaching, safety coaching, is the technique of observing, motivating and fostering safety performance. The current work aims to specifically consider the active ingredients of safety coaching implemented in the offshore oil and gas industry, and, the impact of these on critical safety outcomes. Although the link between coaching and behavioural change has been investigated in an organisational context, the classification of safety coaching is not well defined and safety coaching in safety critical environments is a very under researched area.

\section{Coaching in Safety Critical Environments}

Researchers have noted that many workplace accidents are foreseeable and preventable; the result of a build-up of small mistakes and seemingly unimportant incidents that together can create disaster [3]. The link between the implementation of safety coaching programs and improved workplace safety records has however not been fully demonstrated.

Safety coaching has been defined as:

"Socratic based, future focused dialogue between one individual (safety coach) and a another individual (worker), where the lead individual uses open questions, affirmations, summarises and reflections informed by observation and evidence, aimed at stimulating the self-awareness and personal responsibility of the second individual, with the specific goal of improving safety." [4]

Behavioural based safety coaching can be defined as a process of observation and feedback in order to support safe behaviours and provide constructive feedback on risky behaviours in the workplace [5]. When behavioural based safety coaching was implemented in a large construction firm, positive results were observed including a significant reduction in reported injuries and greater collaboration and care between employees (ultimately increasing safe working behaviours and problem solving) [5]. Arguably, although we have seen improvements in tools and systems producing considerable improvements in organisational safety, many other factors such as attitudes, behaviours and perceptions of risk also impact significantly on producing safe working behaviours. For example, it has been found that driver safety motivation predicted incidents of crashes, and, that driver perceptions of management safety commitment and values also predicted their motivation to engage in safe driving behaviours [6]. This is where safety coaching can play a critical role, engaging employees with safety, setting expectations and creating a sense of responsibility and accountability. It is important for employees to feel empowered and invested in occupational safety. Safety coaching can help to achieve this by promoting the central values of empowerment, emotion and empathy.

Krauesslar, V., Avery, R. \& Passmore, J. (2015). Taking Ownership of Safety. International Journal of Occupational Safety and Ergonomics (JOSE): http://dx.doi.org/10.1080/10803548.2015.1017941. 
The limited research which has explored safety coaching, involving the use of observation and feedback, has been found to reduce error rates in safety critical settings such as radiology hospital departments [7]. Specifically, safety performance has been found to improve with the mean number of days between serious safety events increasing from 200 to 1,031 with no reported incidents [7]. Interestingly, it has also been found that a peer coaching intervention helped to facilitate safe patient handling in a health care setting, with staff reporting increased safety consciousness and confidence in using patient lifting equipment [8]. This is promising in light of the current research purpose because it shows that coaching can be used to facilitate safe working behavioural practices and promote an awareness of safety issues.

Finally, the use of coaching to promote resilient collaboration during the introduction of a new petroleum installation has also been previously investigated [9]. Eight workers involved in the planning of the station took part in five two-and-a-half coaching workshops based on the Coaching for Resilient Collaboration In IO (CORECIO) technique which is designed for high skill level employees. Results indicated that the CORECIO coaching approach helped employees to form an agreed understanding of resilient collaboration and how it should function in the new petroleum installation [9]. Such results illustrate the potential of coaching in forming important principles critical for safe working practices.

\section{Long Term Focus on Safety Culture}

Research also points to the potential long term benefits that safety coaching can provide. In a recent study, two intervention groups of construction site foreman were coached to engage in on-site safety communication with employees [10]. Safety conversations increased dramatically and site safety level improved (impressively an $84 \%$ observed safety level increase in 'railings and coverings') as well as improved safety climate perceptions (improved perceived focus on safety). This research shows how a high risk working environment such as the hazardous, incident prone, construction industry, can improve safety outcomes via coaching, improving safety communication between site managers and employees over time. The similarly high risk oil and gas industry may therefore receive similar safety level improvements through the use of safety coaching focused on promoting changes in safety culture.

The far reaching effects of coaching within the oil and gas industry to produce a complete cultural shift was demonstrated in a coaching program that revolved around being present, visible and accountable for safe working behaviours [11]. The central theme that teams need to work together and unite in creating a safe working environment strongly resembles the 'human factor' initiatives introduced in the airline industry in the 1990's [11]. The results of the coaching program not only included a decrease in incidents across all Norway operations but coaching helped to create a cultural shift within the organisation, making safe behaviour an accepted and expected part of the job. Furthermore, the lasting impact of behaviour grounded safety initiatives (BBS) was 
demonstrated in the introduction of the BBS safety intervention for 11 employees in a research institution [12]. Safety performance increased between the baseline and intervention parts of the research for those experiencing coaching compared to those who were in a control group. Safety performance level for specified safety behaviour variables (such as storing and stacking, sitting posture, smoking) increased from a baseline $74 \%$ to $100 \%$ at the end of the six weeks for the experimental safety intervention group. Employees continued to be observed for their level of safety performance for three months, and the increases in the experimental group were shown to be maintained suggesting that behaviour based safety interventions can produce a lasting impact on safety level performance [12]. The impact of such interventions again encourages the potential of coaching intervention in safety-critical environments.

\section{Coaching and Leadership}

The importance of clear leadership for workplace safety is highlighted by findings that empowering leadership styles are associated with higher compliance and participation with safety by employees and less instances of risky behaviour [13]. However, reports from a safety culture assessment [14] suggest that managers' don't always recognise the effect of poor safety performance on the company as a whole and may be detached from the day to day safety issues. Research has shown that coaching focused on manager-employee relations facilitates safety observations and perceptions [15]. It was found that after a 26 week coaching intervention focused on manageremployee communication meetings, there was a significant increase in the number of safety needs identified and resolved and the level of safety perception also improved [15]. The current research will assess more in depth how this process works in practice, what specific tools and structures are employed by safety coaches and most importantly the perceived results and outcomes of using safety coaching.

In sum, whilst research suggests that coaching is an effective organisational tool used to promote learning and behavioural change there is less evidence that safety coaching specifically works to promote safe working practices and safety outcomes. Therefore this research will investigate just this: how does safety coaching work in practice and what outcomes does it achieve in the, safetycritical, oil industry? A range of experts will be consulted on this and data will be analysed to try and gain a much better understanding of safety coaching and its perceived outcomes.

\section{Current Research}

In light of the literature discussed the purpose of the current research is to address the following questions:

1: Safety coaching purposes and techniques: What practices are safety coaches using and with what objectives in mind? 
2: Achievements of safety coaching: What outcomes are perceived to be achieved as a result of the use of safety coaching application in offshore environments?

3: Future enhancement: How can the performance of safety coaches be further enhanced?

4: Complimentary practices: What other interventions could be employed to achieve safe working in safety critical offshore environments?

\section{Materials and Methods}

\section{Participants}

The 24 participants were recruited from a volunteer sample of expert contacts provided by Embrion consulting. Three separate groups were selected: safety coaches, offshore managers and Health and Safety (HSE) directors. There were 10 safety coaches, 12 offshore managers and 2 HSE directors interviewed. All participants were male. The average length of time that safety coaches had been involved in safety coaching was 4.4 years. For the offshore coaching managers the average length of time managing safety coaching was 9.5 years.

\section{Design and Procedure}

Materials included a semi-structured interview schedule (developed by current authors based on the four research questions detailed above), instructions, debrief and audio recorder. Interviews were conducted over the telephone and lasted between 30 to 40 minutes.

\section{Results}

Audio recordings of interviews were transcribed and then fully analysed using thematic qualitative analysis [16]. Using this analytical approach allowed for emerging themes in and across interviews to be categorised and compared. A number of key themes emerged from the interviews. The most prominent theme identified from both groups was how safety coaching connects and creates an ownership of safety in the individual. Further significant themes revolved around personal significance and humanisation, ingraining safety and assessing and measuring a safety coach's competence. To demonstrate the themes detailed interview quotations and examples have been included and are numbered for example $\mathrm{SC} 1=$ safety coach $1, \mathrm{OCM} 1=$ offshore coaching manager 1 .

\section{Theme 1: Connecting with and creating ownership of safety}

The most prominent theme that emerged was that safety coaching acts to empower people to take ownership of safety themselves. Rather than safety being viewed as an outside issue, as somebody else's concern, safety coaching allows everyone to own and embody safety: 
'Really getting the workforce themselves to start to see safety as something they need to take ownership for' (SC1).

Coaches identified that this is achieved through fostering a strong and consistent safety culture that reaches a deep level of safety values and beliefs. A negative safety culture cannot be changed easily or quickly as at the heart of it is deep rooted and accepted values and beliefs that are embedded in the whole work structure offshore:

'Embedding that new process in the muscle of the platform if you like and then supporting that process to get people in the right place' (SC3).

Both coaches and managers acknowledged the long term sustainability that coaching provides. Empowering ownership of safety to all workers instead of safety being the sole responsibility of safety officials means that it produces a much more effective lasting and sustainable impact. This is because safety is not being directed and ordered, instead, safety coaching creates an ownership culture that approaches safety proactively and independently:

'You're actually fostering it, you're creating the fertile ground to make it grow itself which means that it's more sustainable if you do it that way, you don't have to be on top of things all the time as a manager' (SC1).

Once this successful safety culture is embedded and accepted by the individual as the norm the workforce then become comfortable in questioning, challenging and engaging with safety as it becomes the norm.

\section{Theme 2: Personal significance and humanisation}

Coaches and managers explained that offshore can be inherently detached and disconnected by its very nature. It is a unique and pressurised environment that can cause individuals to react differently to their normal behaviour patterns onshore. It can instead give way to group beliefs that become environmental norms and detach people from their personal ethics. One example recounted by a safety coach revealed just what a powerful tool instilling back that personal significance can be. This coach, after seeing a group of scaffold workers working at height without any safety harness, began asking the group if they knew each other's names, then their children's names, then the names of their wives. This coach discovered that the group knew each other well and that this personal questioning made it salient to them the impact that one of them falling/being injured could have:

'That could be your best mate that guy could live next door to you, your kids could play together' (SC4).

As a result of SC4's intervention he reports that the same team are now taking care, looking out for each other (care over compliance) and wearing the correct safety harnesses. 
Coaches stressed that individuals often feel that they are untouchable, that accidents always happen to other people so therefore safety does not affect them. They noted that the coaching interventions act by humanising and breaking this flawed reasoning down. This suddenly brings it into sharp focus and has been described by one safety coach as a 'light bulb' moment of clarity. OCM2 communicated an instance where the deck crew of the rig displayed a very cavalier attitude towards safety. An intervention was introduced where a safety coach conducted three group sessions with the deck crew to explore where that attitude came from. A wheel task was used to identify where the hazards of the job were located. The workers were encouraged to imagine what it would look, feel and sound like if these dangers were minimized. They were also provided with a pocket book of hazards to refer to. OCM2 then reported that as a result of this the group realised that their own attitudes were contributing to the danger level. The group altered their behaviour patterns so they were acting much more considerately of each other and toolbox safety talks and coaching sessions were consequently taken much more seriously.

OCM 3 expressed concern that the past failings on the offshore industry are not kept fresh in the mind of current offshore workers and complacency can set in as a result. OCM3 suggested that the Piper Alpha video should be shown in safety meetings to bring home to people the devastating effects that a chain of seemingly minor events can cause. He communicated that only by getting people to view tragedy with their own eyes can we make it personally significant to them and their loved ones, to their own lives:

'If we forget it, it could happen again' (OCM3).

\section{Theme 3: Ingraining safety}

The main distinction between advising and coaching was identified by coaches and managers as follows. Safety advising touches a shallow compliance level as opposed to a deeper level used in coaching that can reach someone's values, beliefs and internal norms:

'A coach will unearth a motivation to do the right thing beyond a level of basic compliance' (SC10).

Safety coaching acts to deeply instil safety in the individual, to become part of their thought processes, their concerns:

'We can't get into the heads of people like a safety coach can' (OCM11).

Coaches communicated that safety coaching has to follow a non-directive pattern. It acts to continually raise awareness through interactive discussion, inspiring people to consider their actions and behaviour patterns. This is a subtle gradual process that relies on making the distinction between:

'Intervening and interfering, there's a huge difference' (SC7).

Coaches agree that you have to be able to recognise the successes as well as any failures and gaps in safety. Sometimes this recording of safety behaviours (positive and negative) can be viewed by 
offshore staff as simply superficial recording and it is important to communicate that this is the only way that onshore support staff can keep track of progress. OCM5 communicates that coaches not only act to change behaviour, they also recognise and reinforce good safety performance:

'The change comes from within the person and as a result is much more effective' (OCM2).

Safety coaching encompasses much more than safety, it reaches to the heart of human nature and encourages individuals to evaluate their own attitudes, values and behaviours and what lies behind that.

\section{Theme 4: Assessing and measuring safety coach competence}

The main elevator of an excellent coach was described as their passion and belief in what they are working towards. See Table 1 for an overview of coach qualities identified across the interviews. Coaches need to be motivated by the potential catastrophic consequences that injuries and deaths can cause; they need to be driven to prevent it:

I' $m$ very passionate about what I do because I have seen the effects on families and children of somebody having an accident' (SC4).

'You've got to want to do it and want to make a difference and want to make sure people get home safely' (SC5).

TABLE 1. Qualities of an effective safety coach.

\begin{tabular}{lll}
\hline Personality & Skills & Training/Experience \\
\hline Approachable and friendly. & $\begin{array}{l}\text { Emotional Intelligence: } \\
\text { understanding and interpreting } \\
\text { the subtleties of the situation and } \\
\text { underlying forces of the } \\
\text { organisation. }\end{array}$ & $\begin{array}{l}\text { Experience of offshore industry, time } \\
\text { spent working in different roles. This is } \\
\text { not essential, although it helps to break } \\
\text { down barriers and to build connections } \\
\text { and trust. It helps to build respect and } \\
\text { credibility. }\end{array}$ \\
$\begin{array}{l}\text { Forward thinking, proactive } \\
\text { attitude. }\end{array}$ & $\begin{array}{l}\text { Ability to link together discussions, } \\
\text { to look at the big picture and to } \\
\text { analyse what it means from a } \\
\text { socio-cultural perspective. }\end{array}$ & $\begin{array}{l}\text { Accredited coaching course and } \\
\text { continued refresher training. }\end{array}$ \\
$\begin{array}{l}\text { Communication and } \\
\text { interpersonal skills. }\end{array}$ & $\begin{array}{l}\text { Ability to remain focused, } \\
\text { detached and objective. }\end{array}$ & $\begin{array}{l}\text { Knowledge of different tools, } \\
\text { techniques, behaviours and attitudes. }\end{array}$ \\
$\begin{array}{l}\text { Non-aggressive and able to } \\
\text { build trust and rapport. }\end{array}$ & $\begin{array}{l}\text { Able to hold back and refrain from } \\
\text { being directive and offering their } \\
\text { own experience or suggestions. }\end{array}$ & $\begin{array}{l}\text { Life experience of interacting with a } \\
\text { range of people. }\end{array}$ \\
$\begin{array}{l}\text { Confident, outgoing and } \\
\text { not afraid of conflict. }\end{array}$ & $\begin{array}{l}\text { Identify and work to company's } \\
\text { intentions and aims. }\end{array}$ & $\begin{array}{l}\text { Coaching experience over a number of } \\
\text { years to harness and develop practical } \\
\text { skills. }\end{array}$ \\
Step outside of their & Self-awareness. & $\begin{array}{l}\text { Safety knowledge, for example a } \\
\text { certificate in safety. }\end{array}$
\end{tabular}

Krauesslar, V., Avery, R. \& Passmore, J. (2015). Taking Ownership of Safety. International Journal of Occupational Safety and Ergonomics (JOSE): http://dx.doi.org/10.1080/10803548.2015.1017941. 


\begin{tabular}{|c|c|c|}
\hline $\begin{array}{l}\text { Personal sense of morality } \\
\text { and care for keeping } \\
\text { people safe. }\end{array}$ & Calm under pressure. & Technical knowledge. \\
\hline $\begin{array}{l}\text { Empathy, honesty and } \\
\text { sincerity. }\end{array}$ & Patience and flexibility. & $\begin{array}{l}\text { Report writing experience to deliver } \\
\text { regular feedback to management. }\end{array}$ \\
\hline Non-judgemental. & Explaining and using examples. & $\begin{array}{l}\text { Psychological training, for example } \\
\text { how to approach people. }\end{array}$ \\
\hline Driven and passionate. & $\begin{array}{l}\text { Ability to engage people and to } \\
\text { connect with and pitch ideas to all } \\
\text { areas of the organisation, gaining } \\
\text { support for the need for change. }\end{array}$ & \\
\hline $\begin{array}{l}\text { Personable. } \\
\text { Sense of humour. }\end{array}$ & $\begin{array}{l}\text { Listening and team building. } \\
\text { Able to challenge sensitively and } \\
\text { tactfully. }\end{array}$ & \\
\hline Integrity and humility. & $\begin{array}{l}\text { Recognising that not everyone is } \\
\text { the same, not everyone needs the } \\
\text { same levels of coaching. }\end{array}$ & \\
\hline
\end{tabular}

Safety coaches reported passion and belief as the key drivers of what motivates them to dedicate themselves to such a challenging yet highly influential and rewarding job. It was communicated by several coaches that they have experienced or known of coaches who just communicate at face value and are not truly driven by the need to keep people safe. These are the average coaches. Those that truly encompass safety and believe wholeheartedly in what they are doing set themselves apart into the excellent coach territory, as workers can instinctively tell when a coach is not driven and passionate about their role.

Safety coaches reported questioning and engaging individuals and groups and stimulating safety conversations. By using hypothetical discussions and creating an open atmosphere safety coaches get to the heart of the issues to understand where safety attitudes and values are rooted. Coaching managers' report that coaches encourage workers to see what's happening around them and to work out enhanced ways of carrying out tasks and new approaches to situations:

'By talking to the guys and getting their own fears and aspirations' (SC2).

Safety coaches reported challenging accepted norms that have been maintained and strengthened over the years, providing examples, and most importantly providing reasons why something is important. They stressed the importance of explaining to people why change is necessary, why it is important to carry out the task safely and why there is a healthier way to approach something:

'Explaining to people why intelligent rational human beings get hurt doing the most routine tasks' (SC3). 
Safety coaches conveyed how central it is to facilitate an equal effective interaction of different expertise. This is done by setting an example as a coach, by taking feedback and criticism and being aware of your own limitations as a coach:

'You have to be open to criticism and you have to open to feedback, it's a two-way street and if you don't encourage that two-way street you lose the trust of the guys' (SC2).

Safety coaches described a successful coach's attitude as being objective and approachable and capable of assessing the whole picture first before rushing in with a suggestion. Rather than creating a blame culture the whole system needs to be assessed before devising a plan. This does not mean however that there shouldn't be a strong sense of responsibility and answerability among the workforce:

'For me it's about holding people accountable and responsible to the safety element of the job' (SC9).

OCM4 reports that while he has extremely knowledgeable, highly skilled coaches, some are simply unable to use that knowledge effectively by connecting with people. OCM7 also reports that credibility is a problem for some coaches, with some simply not being natural coaches so proving ineffective:

'You can have all the training you want but if you don't come across right nothing's going to change (OCM6).

Coaching managers reported that by subtly framing language collectively coaches can encourage team investment and perspective change. Both coaches and coaching managers reported the importance of involving people in the safety process. For example OCM7 reports how he has seen effective coaches making it an interactive experience, engaging workers, for example by getting the workers who are actually carrying out the jobs to take part in the risk assessment process.

Theme 5: Role confusion

There is still widespread misunderstanding of safety coaching and SC9 highlighted the need for an industry standard definition or criteria for what a safety coach is, what they are going to do, to help to communicate their value to management:

'It's a chronic lack of understanding about what the term safety coaching actually means, or even coaching in general' (SC1).

Safety coaches report being familiar with an immediate negative reaction of suspicion to any sort of safety role, with it being perceived as simply legality, a box-ticking paperwork role where workers are watched for any deviance:

'The first thing to overcome is the pre thought that you are a safety adviser/officer/policeman. The stigma that these roles carry can turn people off and can often make them nervous when around you' (SC8). 
Safety coaches report that the experience of negative safety coaching, where the coach basically follows a directive safety advisory pattern instead of true behavioural based coaching can lead to mistrust of the role and value of a safety coach. Workers can believe that they are being spied on by management instead of safety being truly valued and paramount within the organisation. Coaches report that they are often met with scepticism from the client as to the perceived worth, need and value of safety coaching interventions. This is due to an industry wide confusion over the role a safety coach and how they are different to an adviser or manager. Often coaching is imposed on contractors so there is some cynicism of its necessity or of its potential:

'As a coach in the safety arena you are often forced upon companies operating for your clients because something has gone wrong' (SC8).

OCM5 communicated an example where a safety coach not only recognised an issue and initiated a new way of working but they actually came up with a more efficient, better way of working that reduced time spent on the job. Coaching is often perceived as a barrier to effective output, however as highlighted by this example it can actually help to produce efficiency gains. Safety coaches distinguished between the safety coach and the more well-known safety adviser role by describing the different processes involved as telling versus listening. Safety advising can mean that people may become dependent on being always told exactly what to do and once this direction stops they will return to their usual behaviour pattern driven by their accepted internalised norms:

'As soon as you turn your back on a work party for example they'll just go back doing what they've always done, they'll resort back to their default behaviour which is directly related to their beliefs' (SC3).

Whilst safety advising and safety coaching were viewed as very distinct they were viewed as complementing each other:

'I don't think you can have one without the other' (SC4).

Many safety coaches also have experience of safety advising and perform mixed roles, with coaching not seen as the primary focus and time allocation of their job. Safety coaching was also viewed as distinct from general or executive coaching. Although similar tools and techniques are employed safety coaching has a very specific agenda, to keep people safe from getting injured or killed, to get them behaving in a safe way. It is therefore:

'Unrealistic to imagine that you can do things exactly the same way as you can if you're doing some executive coaching' (SC1).

\section{Theme 6: Management intervention}

Safety coaches report that part of their job involves communicating to management their part in valuing and promoting the right safety culture offshore, how their actions can impact on how the safety culture is perceived and the resulting work behaviours that it produces: 
'Helping them identify the things that they're doing that will either contribute to people feeling that safety is a priority or not' (SC1).

Safety coaches emphasised the importance of creating unity by involving all areas of the workforce, from the labourer role to the installation manager role. This is achieved by focusing on common aims and commitment, even down to the collective language used. OCM6 reported that coaching can ensure that everyone contributes to the safety of the organisation as a whole, for example by contributing two safety cards per trip to recognise positive and negative safety behaviours or issues. Management can however provide obstacles to workforce ownership of safety by not truly encompassing safety values. This is recognised by workers and undermines the safety culture:

'A lot of management teams don't take ownership themselves' (SC6).

As OCM7 states, management want to see results, they want to know that the money and time they are investing in safety coaching has a proven and measureable outcome. However as OCM7 explains cultural change takes a long time to show and safety statistics take a long time to become obvious and also don't account for severity.

\section{Theme 7: Standardisation}

Many safety coaches and managers reported the industry need for an easily recognisable, standardised coaching qualification. This is not just in house training programmes or specific techniques such as neuro-linguistic programming. Industry standard safety coaching qualifications or criteria would be useful for management teams planning a coaching intervention. This is because:

'Too many companies call people coaches or safety coaches without actually knowing what a safety coach does' (SC9).

Coaches reported that because of this it then creates negative perceptions of safety coaching so that when a true safety coach then turns up he or she is met with resentment and mistrust.

Fundamentally however the individual's personality, attitudes, knowledge and experience was seen as the prevailing factor._Safety coaches reported that training of management teams would also help to create and promote a uniformed strong safety culture. It may also help to introduce a mentoring scheme here where:

'You could identify a safety ambassador that have got a good safety record and they can mentor sort of less experienced managers' (SC9).

Having a mentor to support safety coaches was another suggestion made by safety coaches. It was suggested by SC6 that it may further increase the effectiveness of safety coaches by allowing the coach to work through and make sense of their interactions. Having a confidential contact to debrief, to speak to about what is happening helps to order the coach's own thoughts and provide an additional sense of perspective. This can even be implemented by something as simple as a telephone call to debrief. It was also suggested by SC6 that base level counselling training would be 
beneficial for coaches in order for them to feel confident in dealing with situations where people are opening up about quite personal issues.

\section{Discussion}

This study primarily set out to explore the perceived ingredients and impact of safety coaching implemented in the high-risk offshore industry. The results indicated that safety coaching can create an effective, sustainable long term safety culture within the offshore industry when it is implemented properly and given enough time and support to make a difference. Themes emerged suggesting that safety coaching can promote safe working practices and facilitate positive safety outcomes. The current results are consistent with previous findings [5] that safety coaching resulted in greater collaboration and care between employees as well as safer working patterns. By creating an ownership of safety in the individual, safety can be effectively maintained and managed. Safety coaching can be argued to create empowerment and connection to safety issues. The importance of creating ownership of safety emerged as the most overwhelming theme. All interviewees expressed that safety advising cannot reach this deeper level of safety ownership and empowerment and that this is where safety coaching can prove extremely worthwhile.

Personal significance emerged as a central theme. Personal significance to safety issues awakens the individual, ingraining safety into their personal beliefs and attitudes. A number of coaches recounted examples of where personal meaning has impacted powerfully to provide value and consequence to safety that would otherwise not strike a chord with an individual. Effective safety coaching is however reliant on not only the coach's experience and expertise but more importantly the coach's passion and belief, their ability to inspire and connect with people to make a difference. When asked to select five essential qualities that embody an excellent safety coach, interpersonal communication skills and empathy were consistently prioritised above even training and experience as essential. It was reported that a lack of industry understanding or coaching knowledge could be overcome with experience and effort, whereas empathy and the ability to connect with people are either present or not.

Many coaches and managers highlighted the importance of a coach being able to challenge someone without being confrontational or aggressive and so without the person responding defensively. Safety coaches must embody true approachability and objectivity in order to be trusted and respected by the workforce. Furthermore, safety role confusion is common within the industry, with limited understanding of the role of a safety coach in contrast to the more traditional and accepted safety advisor roles. Consistent with previous research [13] management involvement was also found to be crucial to a successful safety coaching intervention in order to create a consistent, valued safety message. Valuable suggestions were made by coaches and managers in order to further enhance the performance of safety coaches in the future. These included increased priority given to coaching with financial and time resources. This is because many coaches expressed concern that their main role is not viewed as a safety coach, they are also required to fulfil other 
roles such as advising and training, meaning that the majority of their time cannot be spent coaching. Standardisation of safety coaching qualifications and standards was expressed as an important step forward for the industry, helping employers to select appropriate coaches. Tailored training for coaches such as industry matched situational examples as well as mentorship and support was also put forward as a useful advancement. A more long term focus was advocated as an important progression towards creating a more positive, proactive approach to safety within the industry. This is in contrast to the current short term reactive incident led approach in response to safety lapses. Coaches and managers report that this process of change is beginning to take place slowly within the industry, although there is still a long way to go for it to become accepted and recognised.

\section{Conclusion}

The current work has found that the use of safety coaching in the offshore oil and gas industry is a powerful tool in managing and promoting a culture of safety and care. By accessing peoples' personal beliefs and intrinsic motivation, coaches can act to stimulate personal reflection and encourage behaviours which ensure greater consistency between values and actual behaviours. Safety coaching should be seen as an important element for building a safety culture, but to achieve these outcomes coaches need to be trained and competent in coaching skills, as well as having an awareness of safety issues facing offshore workers. Future research would benefit from gathering the views and experiences of those receiving the coaching, as this is a limitation of the current study, which was confined to coaches and managers. It would be useful to find out how those receiving safety coaching feel such coaching has affected them and what they perceive to be its value within the industry.

\section{References}

Alamgir H, Drebit S, Guiyun H, Kidd C, Tam H, Fast C. Peer Coaching and Mentoring: A New Model of Educational Intervention for Safe Patient Handling in Health Care. American Journal of Industrial Medicine. 2011; 54: 609-617.

Al-Hemoud A, Al-Asfoor M. A behaviour based safety approach at a Kuwait research institution. Journal of Safety Research. 2006; 37: 201-206.

Braun V, Clarke V. Using Thematic Analysis in Psychology. Qualitative Research in Psychology. 2006; 3(2): 77-101 
Dickerson J, Koch B, Adams J, Goodfriend M, Donnelly L. Safety coaches in radiology: decreasing human error and minimizing patient harm. Pediatric Radiology. 2010; 40: 1545-1551.

Geller E, Perdue S, French A. Behavior-Based Safety Coaching 10 guidelines for successful application. Professional Safety. 2004: 42-49.

Grant A, Passmore J, Cavagh M, Parker H. The state of play in coaching. International Review of Industrial and Organizational Psychology. 2010; 25: 125-168.

Kines P, Andersen D, Andersen L, Nielsen K, Pedersen L. Improving safety in small enterprises through an integrated safety management intervention. Journal of Safety Research. 2010; 44: 87-95.

Kines P, Anderson L, Spangenberg S, Mikkelsen K, Dyreborg J, Zohar D. Improving construction site safety through leader-based verbal safety communication. Journal of Safety Research. 2010; 41(5): 399-406.

Martinez-Corcoles M, Gracia F, Tomas I, Peiro J, Schobel M. Empowering team leadership and safety performance in nuclear power plants: A multilevel approach. Safety Science. 2013; 51: 293-301.

Mathis T. Supervisory Safety Coaching: Growing a Safety Culture from the Middle Out. EHSToday. 2009: 20-22. Retrieved July 2013, from: http://ehstoday.com/safety/supervisory-safetycoaching-culture-5634.

Newnam S, Griffin M, Mason C. Safety in Work Vehicles: A Multilevel Study Linking Safety Values and Individual Predictors to Work-Related Driving Crashes. Journal of Applied Psychology. 2008; 9(3): 632-644.

Olivero G, Bane K, Kopelman R. Executive Coaching as a Transfer of Training Tool: Effects on Productivity in a Public Agency. Public Personnel Management. 1997; 26(4): 461-469.

Passmore J, Fillery-Travis A. A critical review of executive coaching research: a decade of progress and what's to come. Coaching: An International Journal of Theory, Research and Practice. $2011 ; 4(2)$ : 70-88.

Pate-Cornell E. Learning from the Piper Alpha Accident: A Postmortem Analysis of Technical and Organizational Factors. Risk Analysis. 1993; 13(2): 215-232.

Renning S. Innovative coaching program enhances safety culture. Offshore. 2007: 80-81.

Skerve A, Kaarstad M, Storseth F, Waero I, Grotan T. Planning for resilient collaboration at a new petroleum installation - A case study of a coaching approach. Safety Science. 2010; 50: 1952-1959. 